\title{
Development of filial attachment to static visual features of an imprinting object
}

\author{
LEONARD A. EISERER \\ Franklin and Marshall College, Lancaster, Pennsylvania 17604
}

\begin{abstract}
Three experiments investigated the fact that the static visual features of an imprinting object (its color, size, and shape characteristics, as represented by the object remaining stationary during its presentation) can gradually acquire control over filial behavior by virtue of their repeated spatial-temporal pairing with visual motion-an effect that has been interpreted as a process of classical conditioning. Experiment 1 found that the static features can acquire control if they are made conspicuous in some other, nonmovement way (i.e., by manipulating the relative illumination of the imprinting object). Further experiments then failed to find any qualitative difference between the behavioral control developed by conspicuous but static aspects of a duckling's environment and the control developed by the static features of a moving object, either in terms of persistence of the acquired control (Experiment 2) or in terms of possible restrictions imposed by the sensitive period for imprinting (Experiment 3). Taken together, these results support the plausibility of a perceptual learning hypothesis, although the classical conditioning view was not itself contradicted. The present findings are also relevant to the broader issue of whether any valid distinctions exist between the behavioral control exerted by static aspects of a precocial bird's environment and the control exerted by animate, presumably social stimuli.
\end{abstract}

When an arbitrarily selected object is initially presented in motion to a newly hatched duckling, that object very rapidly elicits filial behavior from the duckling (Hoffman, Stratton, Newby, \& Barrett, 1970). Although the duckling may not initially direct filial responses toward the object if it remains stationary during its presentation (Hoffman et al., 1970 ), the young bird will do so after receiving prolonged exposure to the object in motion (Eiserer, 1977; Eiserer \& Hoffman, 1973). Importantly, the acquisition of behavioral control by the stationary imprinting object can be demonstrated even under conditions where prolonged exposure to the stationary object itself is not sufficient for the acquisition to occur (i.e., when repeated exposure to the object in motion is essential; Hoffman, Eiserer, \& Singer, 1972).

It should be realized that presentation of a stationary imprinting object is the equivalent of presenting all of the static visual features of that object-namely, its particular color, its particular size, and its particular shape. The above-cited research, then, indicates that such static visual features-at least with the kinds of biologically unnatural objects that are used

This research was supported in part by a grant from the Mellon Foundation. The author would like to thank Douglas S. Ramsay and Donna S. Lansman for their help in conducting these experiments. This manuscript benefited greatly from the comments of Animal Learning \& Behavior reviewers and editors. Reprint requests should be sent to the author at Whitely Psychological Laboratories, Franklin and Marshall College, Lancaster, Pennsylvania 17604. in many imprinting experiments-are essentially neutral in their initial effects upon filial responses, but gradually acquire behavioral control as a young bird is exposed to the object in motion. This acquisition of behavioral control by the initially neutral features of an imprinting object is a fundamentally important phenomenon, as it would seem to represent the very essence of the development of infantile attachments to specific surrogate objects. It is, after all, the particular color, size, and shape of any given object that make it discriminably different (at least visually) from other objects.

Moreover, the acquisition phenomenon is also of great theoretical interest, for it is one of the empirical focal points of the classical conditioning theory of imprinting (Hoffman \& DePaulo, 1977; Hoffman \& Ratner, 1973a). According to this view, visual motion constitutes an unconditioned stimulus (US) that innately elicits filial behavior from newly hatched ducklings and then gradually bestows this behavioral control onto initially neutral stimuli (the object's color, size, or shape characteristics) with which it is associated. Consistent with this interpretation is the fact that the initial elicitation of filial behavior by a moving object is so immediate as to suggest the operation of an unlearned reaction (i.e., an unconditioned response or UR) to the stimulation provided by the motion of the object (Hoffman et al., 1970). Additional supportive evidence comes from the clear demonstration by Hoffman, Barrett, Ratner, and Singer (1972) that classical conditioning of filial responses can occur in a conventional 
Pavlovian paradigm-namely, the forward pairing of a light stimulus with an imprinting object.

It should be noted, however, that exposure to an inprinting object typically occurs under conditions that do not precisely parallel the conventional Pavlovian situation, inasmuch as the former entails simultaneous pairing of the conditioned stimulus (CS) and US (i.e., the duckling is exposed to the initially neutral features at the same time that it is exposed to the stimulation provided by visual motion), rather than the traditional Pavlovian procedure of forward pairing. Imprinting also involves a situation in which the CS and the US reside within a single stimulus complex (i.e., the static visual features and the visual motion are components of one and the same object), whereas conventional Pavlovian procedures usually entail physically discrete stimuli (e.g., a bell and meat powder). In this respect, the imprinting situation may more closely resemble the learning of sights and smells associated with particular foods. As Pavlov (1927) noted, "the effect of sight and smell of food is not due to an inborn reflex, but to a reflex which has been acquired in the course of the animal's own individual existence.... Only after the puppies have been allowed to eat bread and meat on several occasions does the sight or smell of these foodstuffs evoke the (salivary) secretion" (p. 22). This example resembles the imprinting situation because, in both cases, the functionally neutral aspects of a stimulus complex acquire control over behavior that is initially elicited only by the complex as a whole.

One alternative to the classical conditioning hypothesis can be derived from the perceptual learning theory that has been described by numerous imprinting researchers (Bateson, 1966; Brown \& Hamilton, 1977; Salzen, 1962; Sluckin \& Salzen, 1961). Rather than serving as a US in the Pavlovian sense, the innate releaser of visual motion may merely serve to make the static features of the imprinting object very conspicuous ${ }^{1}$ relative to other aspects of the duckling's environment. Because of this increased conspicuousness, the duckling may attend to the features more, and hence become more familiar with those features than with other aspects of its immediate environment. Granting this enhanced familiarity, then, one need only assume that young precocial birds will show filial-type responses to stimuli that are familiar to them, an assumption that enjoys empirical support (Malcolm \& Graves, 1977).

The present series of experiments further investigated the plausibility of perceptual learning theory as an explanation for the acquisition of behavioral control by static visual features of an imprinting object. If visual motion exerts its effects by simply increasing the conspicuousness of an object's static visual features, then it should be possible to mimic the effects of movement by making those features conspicuous through some other, nonmovement means. Experiment 1 tested this possibility by exposing one group of ducklings to a stationary light stimulus in a brightly illuminated environment (the light stimulus thus being relatively inconspicuous), and exposing a second group to the same stationary stimulus in a dimly illuminated environment (the light stimulus thus standing out like a beacon); for purposes of comparison, a third group of subjects was exposed to the light stimulus in motion and within a brightly illuminated environment. Perceptual learning theory would predict the development of strong attachment to the static visual features in those groups for which such features were made conspicuous (i.e., the second and third groups) but not in the group for which the features were little more conspicuous than other aspects of the surrounding environment (the first group).

\section{GENERAL METHOD}

\section{Subjects}

Experiments 1 and 3 in the present series each used a new group of Khaki Campbell ducklings (Anas platyrhynchos domesticus) that were hatched in visual isolation from eggs obtained from George F. Shaw, Inc., West Chester, Pennsylvania. As will be described later, the subjects in Experiment 2 were the same as those used in Experiment 1.

Except for periods spent in the experimental apparatus, each duckling was maintained in an individual housing unit consisting of a brown rectangular box $(30 \times 51 \times 30 \mathrm{~cm})$ that was partially filled with bedding material. Under these circumstances, the ducklings could hear each other but their visual environment was restricted to that provided by the inside of their individual housing units. While in those units, the ducklings had continuous access to food and water.

\section{Apparatus}

The experimental apparatus consisted of a plywood box $(120 \times$ $66 \times 78 \mathrm{~cm}$ ) divided by a fine-mesh stainless steel screen into two compartments, one for the duckling $(84 \times 66 \times 78 \mathrm{~cm})$ and the other for the imprinting object $(36 \times 66 \times 78 \mathrm{~cm})$. The imprinting object consisted of three circular lights (one red, one green, and the third blue) mounted vertically on a wood panel that moved horizontally along the length of the stimulus compartment. These lights were each $1.2 \mathrm{~cm}$ in diameter and spaced some $2.5 \mathrm{~cm}$ apart from each other. Presentations of the moving stimulus were produced by illuminating the three colored lights and moving the wood panel back and forth across the stimulus compartment at approximately $5.3 \mathrm{~cm} / \mathrm{sec}$. Presentations of the stationary stimulus were produced by illuminating the three colored lights while withholding power from the motor that moved the wood panel. Complete stimulus withdrawal was accomplished by extinguishing the colored lights and, if necessary, stopping the movement of the wood panel.

To permit assessment of the ducklings' locomotor behavior during testing, the carpeted subject compartment was divided into two unequal portions by a strip of adhesive tape running parallel to, and at a distance of $17 \mathrm{~cm}$ from, the fine-mesh screen of the stimulus compartment. Hence, the resulting small and large areas of the subject compartment were 17 $\times 66 \mathrm{~cm}$ and $67 \times 66 \mathrm{~cm}$, respectively, with the smaller area (the "approach area") nearest the stimulus compartment. A second section of fine-mesh screen (i.e., in addition to the screen 
that separated the subject and stimulus compartments) was built into one of the side walls of the subject compartment. This screen ( $36 \mathrm{~cm}$ high $\times 80 \mathrm{~cm}$ long) permitted the experimenter to observe the exact location of a duckling while it was in the imprinting apparatus.

Lighting in the subject compartment was provided by two continuously illuminated incandescent lamps mounted along the top of the compartment. These lamps were positioned so that, unless the imprinting stimulus lights were also illuminated, the light which reflected from the fine-mesh screen prevented the subject from seeing into the darkened stimulus comparment. The same principle operated to prevent subjects from seeing the human observer as well.

\section{EXPERIMENT 1}

\section{Method}

Subjects. Thirteen Khaki Campbell ducklings were used.

Procedure. At $10-16 \mathrm{~h}$ posthatch, each duckling was assigned to one of three experimental groups: an inconspicuous stationary stimulus (ISS) group $(\mathrm{N}=4)$, a conspicuous stationary stimulus (CSS) group ( $N=4)$, and a moving stimulus (MS) group $(\mathrm{N}=5)$. Although these $\mathrm{N}$ sizes are small relative to those typically employed in the imprinting literature, previous, closely related research (Eiserer, 1977; Eiserer \& Hoffman, 1974; Eiserer, Hoffman, \& Klein, 1975; Hoffman, Eiserer, \& Singer, 1972) has indicated that the phenomenon of concern is robust enough to bear investigation with a small- $\mathrm{N}$ methodology. Whenever feasible, small sample sizes would seem a preferred research strategy because of the obvious savings in research time, equipment wear, and animal lives.

During exposure sessions for ducklings in the ISS group, the lights in the subject compartment were made very bright (i.e., 100-W bulbs were used) and the imprinting stimulus lights remained illuminated but stationary in the center of the stimulus compartment. During exposure sessions for ducklings in the CSS group, the lights in the subject compartment were made very $\operatorname{dim}$ (7.5-W bulbs) and the imprinting stimulus lights remained illuminated but stationary. During exposure sessions for ducklings in the MS group, the lights in the subject compartment were kept very bright (100-W bulbs) and the illuminated imprinting stimulus lights were kept in continuous motion.

All ducklings received four 15 -min exposure sessions at the rate of two sessions per day; the first and second sessions began at 10-16 $\mathrm{h}$ and 18-24 $\mathrm{h}$ posthatch, respectively. Immediately following each of the four sessions, as well as immediately before the very first session, each duckling received an approach test, the stimulus conditions of which were identical for all three groups. More specifically, the subject compartment lights were kept at bright illumination $(100-\mathrm{W})$ and the illuminated imprinting stimulus lights remained stationary during their presentation. At the start of a given test, the imprinting stimulus remained withdrawn for $60 \mathrm{sec}$, then was presented for $60 \mathrm{sec}$, then withdrawn for another $60 \mathrm{sec}$, and finally presented again for $60 \mathrm{sec}$. In order to ensure that the duckling had to make an active response to accumulate time in the approach area near the imprinting stimulus, the experimenter placed the bird near the center of the subject compartment at the beginning of each of the above four 60 -sec intervals.

The experimenter monitored the position of the duckling throughout the tests as well as during a representative sample of exposure sessions. Approach was defined as the number of seconds that the duckling spent within the area of the subject compartment near the stimulus, beginning as soon as any portion of the bird's foot touched the strip of tape that bordered the area.

\section{Results}

By the end of the four exposure sessions, three of the four ducklings that were presented with the conspicuous stationary stimulus developed a strong approach tendency to that stimulus; this tendency was not only evident during the exposure sessions themselves, but persisted even when the birds were tested under bright houselights. Four of the five ducklings that were exposed to the moving stimulus under bright houselights also developed a strong approach response to the stationary stimulus (the fifth subject actually failed to approach the stimulus even when it was moving, but this bird appeared to have a slight equilibrium problem during the sessions and, in fact, died shortly after the experiment ended). In contrast, none of the birds that were exposed to the inconspicuous stationary stimulus approached the stimulus with any regularity.

These effects are reflected in Figure 1, which shows the mean seconds of approach to the stationary stimulus (i.e., during its two $60-\mathrm{sec}$ presentations in the approach tests) by the three groups of subjects, as a function of duration of exposure. The figure reflects the data of all subjects, including those birds in the MS and CSS groups that failed to approach. An analysis of variance (two-factor mixed design: repeated measures on one factor) yielded a marginally reliable overall effect of exposure condition $[F(2,10)=3.29, p<.09]$, a significant overall effect of length of exposure $[F(4,40)=4.48, p<.01]$, and most importantly, a significant interaction between exposure length and exposure condition $[F(8,40)=4.60, p<.01]$.

\section{Discussion}

The presently found difference between ducklings exposed to the moving stimulus and those exposed to the inconspicuous stationary stimulus replicates the finding of Hoffman, Eiserer, and Singer (1972) that the static visual features of an animate imprinting object can gradually acquire the ability to elicit filial behavior, even under conditions where pro-

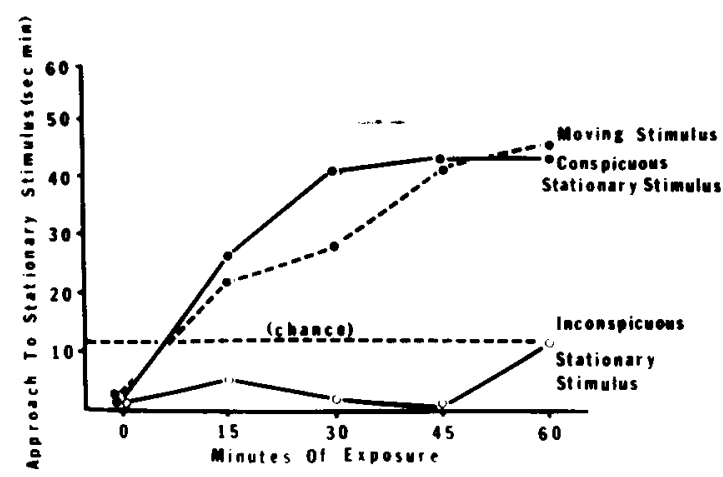

Figure 1. Mean seconds of approach to the stationary stimulus as a function of exposure duration, for newly hatched ducklings presented with the moving stimulus, the conspicuous stationary stimulus, or the inconspicuous stationary stimulus. Since the approach area was approximately $20 \%$ of the total area of the subject compartment, the "chance" level of approach was considered to be $12 \mathrm{sec} / \mathrm{min}$. 
longed exposure to those features by themselves is insufficient for the development of such control. However, the results of Experiment 1 also clearly show that newly hatched ducklings can develop strong approach tendencies towards stationary stimuli in their environment, so long as those stimuli are very conspicuous. Previous authors have already documented the occurrence of imprinting to stationary stimuli (Bateson, 1964c; Gray, 1960; Malcolm \& Graves, 1977; Salzen, Lily, \& McKeown, 1971; Taylor \& Taylor, 1964), as well as the importance of stimulus conspicuousness in imprinting (Bateson, 1964c; Gray, 1960). The present findings, in turn, suggest that the primary role of visual movement in the context of imprinting may only be to make certain objects conspicuous against the background of an otherwise static environment.

It might be possible for a classical conditioning theorist to suggest that conspicuousness per se can constitute a US for filial behavior, and that in the present study the relatively inconspicuous features of the stimulus (its size and shape, for example, as opposed to its bright color) served as CSs for ducklings in the CSS group. There is, however, the theoretical pitfall of postulating an ever-expanding list of potential USs in the context of imprinting (such a list would probably already include visual flicker, naturalistic auditory stimuli, and perhaps certain tactile stimuli as well as visual movement). Thus, a perceptual learning interpretation of the present findings would seem more parsimonious than one involving classical conditioning.

\section{EXPERIMENT 2}

As noted earlier, previous work (Eiserer \& Hoffman, 1974; Hoffman, Eiserer, \& Singer, 1972) has indicated that, at least under certain conditions, visual motion is critical to the development of filial attachment to initially neutral features of an imprinting object. It has also been found, however, that such attachment-after it has developed via the operation of visual movement-does not weaken or "extinguish" if visual movement is subsequently withheld and the subjects receive prolonged exposure to the features by themselves (Eiserer, Hoffman, \& Klein, 1975; Gaioni, Hoffman, DePaulo, \& Stratton, 1978). In short, once the initially neutral features acquire behavioral control, that control subsequently persists even under conditions that were insufficient for the initial acquisition.

Experiment 2 asked whether this marked persistence would also characterize the behavioral control developed by a conspicuous, but stationary stimulus. Using subjects from the CSS group of Experiment 1, the present study sought to determine whether the approach response would weaken if the ducklings received prolonged exposure to the stationary lights when they were kept inconspicuous-that is, under conditions that had been insufficient for development of the approach response in the first place. For purposes of comparison, the persistence of the approach response of ducklings in the MS group of Experiment 1 was also assessed under similar conditions (namely, across a period of prolonged exposure to the inconspicuous stationary stimulus).

\section{Method}

Subjects. The present experiment employed four of the ducklings that had been in the MS group of Experiment 1, and two of the ducklings that had been in the CSS group. These six ducklings were selected on the basis of the strong approach responses they had developed towards the stationary stimulus (an additional subject in the CSS group had also approached consistently, but this bird died shortly after Experiment 1).

Procedure. Beginning on the day following completion of Experiment 1, each of the six ducklings received a series of 40 15-min extinction sessions at the rate of three sessions per day (the intersession interval within any given day was approximately $2 \mathrm{~h}$ ). Each session simply consisted of placing the duckling in the apparatus and presenting the stationary stimulus lights for 15 continuous minutes, with the lights in the subject compartment at bright illumination $(100-W)$. At the end of each session, the duckling was given an approach test that was identical in procedure to the tests given in Experiment 1 (i.e., approach to the stationary stimulus was assessed with the subject compartment lights at bright illumination). At no point during Experiment 2 was any sibject ever exposed to visual movement, nor were the subject compartment lights ever dimmed.

\section{Results}

Observations during a random sample of the exposure sessions indicated that each duckling in both groups remained very close to the stationary stimulus throughout the entire course of the experiment. Each duckling also showed nearly immediate approach to the stimulus during each of the 40 tests, the lone exception being one bird in the MS group that failed to approach the stimulus at all in Test 2. Figure 2, which shows the mean seconds of proximity for both groups of ducklings, confirms that the subjects' filial behavior gave no indication of weakening across the 40 tests. Although the present results do not permit the conclusion that the acquired approach response

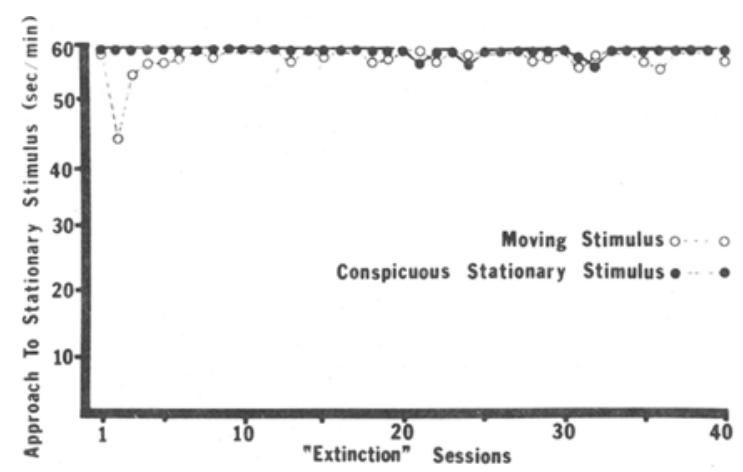

Figure 2. Mean seconds of approach to the stationary stimulus as a function of extinction sessions, for ducklings that had been previously exposed to either the moving stimulus or to the conspicuous stationary stimulus. During extinction sessions, subjects were exposed to the inconspicuous stationary stimulus. 
would have persisted indefinitely, they do indicate that the response persists for an extremely long time. After all, the approach response remained at asymptotic strength after an amount of extinction exposure (40 15-min sessions, or a total of $600 \mathrm{~min}$ ) that was 10 times the amount of exposure needed for the response to be acquired initially (refer to Figure 1).

\section{Discussion}

The presently found persistence of the acquired approach response in the MS group replicates earlier work (Eiserer et al., 1975; Gaioni et al., 1978), and at first thought might seem to be inconsistent with classical conditioning theory. According to this theory, the sessions of exposure to the stationary stimulus represented repeated presentations of the CS without further pairings with the US; in conventional Pavlovian conditioning, such a procedure generally leads to relatively quick extinction of the acquired response. However, there are certain contexts in which conditioned responses can sometimes be particularly resistant to extinction-such as an acquired taste aversion (Garcia, Kimeldorf, \& Koelling, 1955)-and it is possible that imprinting simply represents another case of unusually extinctionresistant learning. It is also possible that the peculiar nature of the association between the supposed CS and US (i.e., the fact that both reside within the same stimulus complex, and that their temporal pairing is simultaneous) underlies the unusual resistance to extinction.

From the viewpoint of perceptual learning theory, the nonextinction effect poses no problem whatsoever. Once a conspicuous stimulus has been rendered familiar to a duckling, there is no way it can subsequently become "unfamiliar" (i.e., familiarization is logically an irreversible process, at least barring some sort of memory deficit). Thus, visual movement should be superfluous once the acquisition process has occurred.

In any event, the present study failed to detect any difference in persistence between birds that formed an attachment to the static characteristics by virtue of the latter's pairing with visual motion and birds that formed such an attachment by virtue of the characteristics being made conspicuous through means other than visual motion. This lack of difference is consistent with the notion that no qualitative distinction exists between the two means of acquisition-i.e., that they both represent essentially the same process.

Experiment 3 provided another opportunity to detect a qualitative difference between the two acquisition procedures. Many researchers (Baron \& Kish, 1960; Bateson, 1964a; Boyd \& Fabricius, 1965; Brown, 1975; Hoffman, Ratner, \& Eiserer, 1972) have demonstrated that precocial birds can form attachments to a novel moving object even if their initial exposure to the object occurs later than the 1st day posthatch (i.e., after the sensitive period). Experiment 3 sought to determine whether ducklings could also form an attachment to a conspicuous, but static, stimulus after the end of the sensitive period. As such, the study offered a test of the generality and replicability of the findings of Experiment 1.

\section{EXPERIMENT 3}

\section{Method}

Subjects. A total of 13 newly hatched ducklings served as subjects. The birds were obtained from two different hatches of 9 and 4 ducklings each (see below).

Procedure. The first nine ducklings were transferred from the incubator to individual housing units at approximately $12 \mathrm{~h}$ posthatch. The birds remained in these units until Day 5 posthatch, whereupon they were randomly divided into two groups: a MS group $(\mathbb{N}=4)$, which was exposed to the moving stimulus lights while the lights in the subject compartment were at bright illumination $(100-W)$, and a CSS group $(N=5)$, which was exposed to the stationary stimulus lights while the subject compartment lights were at dim illumination $(7.5 \mathrm{~W})$. The four ducklings in the second hatch were also transferred from the incubator to individual housing units at $12 \mathrm{~h}$ posthatch. They remained in these units until Day 5 posthatch, whereupon they were assigned to the ISS group ( $\mathrm{N}=4)$, which was exposed to the stationary stimulus lights while the subject compartment lights were at bright illumination ( $100 \mathrm{~W})$.

All ducklings received seven 15 -min exposure sessions at the rate of two sessions per day. Immediately following each of the sessions, as well as immediately before the very first session, each duckling received an approach test with the stationary stimulus. Each test was identical in procedure to the tests given in Experiments 1 and 2

\section{Results}

During the first one or two exposures, ducklings in the MS group showed fear-type responses (distress calling, avoidance responses, etc.) towards the imprinting stimulus. Such behavior waned rather quickly, however, and all four of the birds then shifted from fear responses to filial ones: They ceased giving distress calls during presentaton of the stimulus, and, instead of avoiding proximity, they actually spent most of each session in the area nearest the fine-mesh screen. At this point, they were clearly attending to the stimulus and, in fact, often followed it back and forth. Similar effects characterized the behavior of the five ducklings in the CSS group, with the qualifications that (1) less vigorous fear responses appeared during the initial exposure sessions, and (2) only three of the five birds ever developed strong approach tendencies (the remaining two subjects approached an average of $23 \mathrm{sec} / \mathrm{min}$ during the last test).

Ducklings in the ISS group gave a large number of distress calls during the first few exposure sessions, but their high rate of locomotor behavior was 


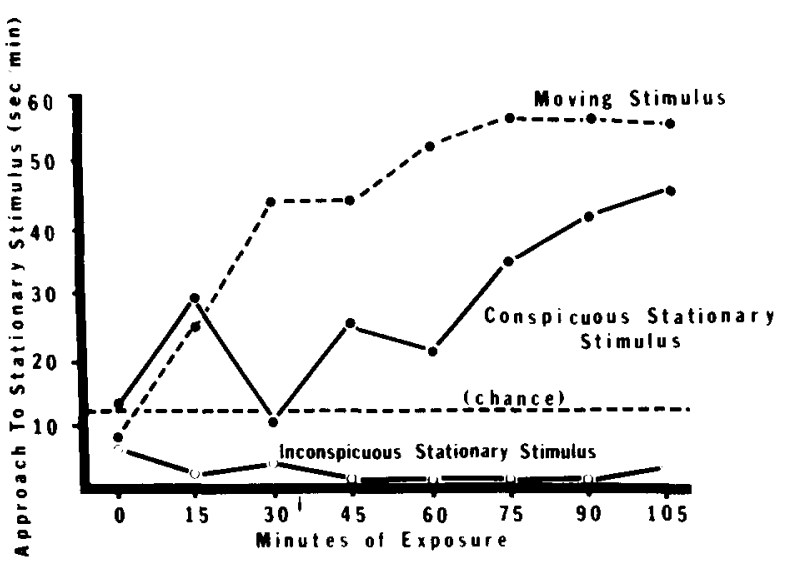

Figure 3. Mean seconds of approach to the stationary stimulus as a function of exposure duration, for 5-day-old ducklings presented with the moving stimulus, the conspicuous stationary stimulus, or the inconspicuous stationary stimulus.

generally random and could not be characterized as oriented either towards or away from the stimulus lights specifically. Instead, it was as if these birds found the whole experimental environment aversive. As the ducklings received more and more sessions in the apparatus, their distress calling ceased almost entirely and they spent a large portion of each session sitting quietly in the subject compartment.

Figure 3 shows, for each group of subjects, the mean seconds of approach to the stationary stimulus as a function of duration of exposure. Analysis of variance (two-factor mixed design: repeated measures on one factor) revealed a significant overall effect of exposure condition $[\mathrm{F}(2,10)=7.45, \mathrm{p}<.02]$, a significant overall effect of length of exposure $[F(7,70)=5.63, p<.01]$, and a significant interaction between exposure condition and exposure length $[F(14,70)=3.42, p<.01]$. These results, then, provide a successful replication of the basic findings of Experiment 1.

\section{Discussion}

In the present study, ducklings in the MS group initially showed fear towards the imprinting stimulus, but with continued exposure this fear declined and was actually supplanted by positive approach responses. This fear-then-filial pattern represents a commonly found phenomenon in precocial birds that are exposed to a novel moving object after the sensitive period (Bateson, 1964a; Hoffman, Ratner, Eiserer \& Grossman, 1974; Salzen, 1962). As has been noted elsewhere (Eiserer, 1978; Hinde, 1955; Hoffman \& Ratner, 1973a), such a finding argues against the once-popular conception of a strict critical period for imprinting. Of more importance for present purposes, however, is the finding that a similar, gradual attachment process occurred in the CSS group as well. True, the ducklings' initial fear towards the novel static stimulus appeared to be less intense than their fear towards the novel moving stimulus (a finding previously reported by Bateson, $1964 \mathrm{c})$, and their rate of attachment to the static stimulus was slower than it was to the moving stimulus (refer to Figure 3). But these differences are merely quantitative rather than qualitative, and thus do not imply a basic distinction in process between the two modes of attachment. This conclusion is, in turn, consistent with the implication of Experiments 1 and 2 that a primary role of visual movement in imprinting may simply be to ensure a quality of conspicuousness for selected stimuli in a young bird's environment.

\section{GENERAL CONCLUSIONS}

The present series of experiments failed to find any qualitative difference between the behavioral control developed by conspicuous, but static, aspects of a duckling's environment and the control developed by the initially neutral features of a moving imprinting object. In both cases, the control over approach responses develops gradually (at least under the presently employed testing procedures) when ducklings are given exposure that begins during the 1st day posthatch (Experiment 1); in both cases, the control-once it has developed-is maintained even if the ducklings subsequently receive prolonged exposure under conditions that were not sufficient for the initial development of that control (Experiment 2); and in both cases, the control can develop even if the initial exposure does not occur until well past the end of the sensitive period (Experiment 3 ).

These findings seem related to the broader issue of whether any valid and fundamental distinctions exist between the behavioral control exerted by static aspects of a young precocial bird's environment and the control exerted by animate, presumably social stimuli. Indeed, many impressive similarities exist between these two categories of attachment. First, feeding behavior is facilitated both by the presence of a moving imprinting object (Graves, 1973; Hoffman, Stratton, \& Newby, 1969) and by the presence of familiar static stimuli (Jones, 1977). Second, neither familiarity with a given static environment nor familiarity with a given moving stimulus is sufficient for newly hatched birds to begin to exhibit fear of novelty (i.e., presumably some amount of physiological maturation is also needed; Hoffman \& Ratner, 1973b). Third, precocial birds that are at least a few days old show the same sort of fear behavior (crouching, distress calls, flight) in response to novel static settings that they do in response to novel moving objects (Hoffman, Ratner, \& Eiserer, 1972; Malcom \& Graves, 1977; Salzen, 1962; Experiment 3 in the present series). Fourth, just as a fear stimulus will induce a young precocial bird to more strongly approach a familiar moving 
object (Sluckin \& Salzen, 1961), so too will it induce approach to a manipulable, but otherwise static, object with which a bird has been reared (Candland, Nagy, \& Conklyn, 1963). Finally, the finding by Bateson (1964b) that birds imprint to a moving object more readily if it is marked with the same distinctive visual pattern as are the walls of the birds' housing units implies a commonality of process in the formation of attachment to static environmental and moving social stimuli.

It is, in fact, difficult to point to any clear distinction between the attachments that precocial birds form to a moving object and to the static environment with which they become familiar. Salzen (1970) has suggested one possible distinctionnamely, that contact reinforcement (tactile stimulation) may be "necessary for fixation of a social attachment, i.e., for imprinting, but not for persistent performance of approach and following" to a familiar aspect of the environment (p. 170)-but the evidence for this hypothesis remains inconclusive. Hoffman and Ratner (1973b) found that silent ducklings in a familiar environment began emitting distress calls after a moving stimulus was briefly presented and then withdrawn, implying a lack of complete equivalence between the effects of a moving stimulus and those of familiar, but static, stimuli. This difference, however, may merely represent a quantitative distinction (i.e., that moving stimuli are more powerful in their behavioral control) rather than a truly qualitative one. In summary, then, there seem to be many more similarities than differences between the attachments that young precocial birds form to animate objects and those they form to inanimate aspects of their surrounding environment.

\section{REFERENCES}

Baron, A., \& Kish, G. B. Early social isolation as a determinant of aggregative behavior in the domestic chicken. Journal of Comparative and Physiological Psychology, 1960, 53, 459-463.

Bateson, P. P. Changes in chicks' responses to novel moving objects over the sensitive period for imprinting. Animal Behaviour, 1964, 12, 479-489. (a)

Bateson, P. P. Effect of similarity between rearing and testing conditions on chicks' following and avoidance responses. Journal of Comparative and Physiological Psychology, 1964, 57, 100-103. (b)

Bateson, P. P. Relation between conspicuousness of stimuli and their effectiveness in the imprinting situation. Journal of Comparative and Physiological Psychology, 1964, 58, 407-411. (c)

Bateson, P. P. The characteristics and context of imprinting. Biological Reviews, 1966, 41, 177-220.

Boyd, H., \& Fabricius, E. Observations on the incidence of following of visual and auditory stimuli in naive mallard ducklings (A nas platyrhynchos). Behaviour, 1965, 25, 1-15.

Brown, R. T. Following and visual imprinting in ducklings across a wide age range. Developmental Psychobiology, 1975, 8, 27-33.

Brown, R. T., \& Hamilton, A. S. Imprinting: Effects of discrepancy from rearing conditions on approach to a familiar imprinting object in a novel situation. Journal of Comparative and Physiological Psychology, 1977, 91, 784-793.

Candland, D. K., Nagy, Z. M., \& Conklyn, D. H. Emotional behavior in the domestic chicken (White Leghorn) as a function of age and developmental environment. Journal of Comparative and Physiological Psychology, 1963, 56, 1069-1073.

Eiserer, L. A. Behavioral control by stimulus components of an imprinting object. Animal Learning \& Behavior, 1977, 5, $153-160$.

Eiserer, L. A. Anomalous effects in psychology: Parallels between the brain stimulation and imprinting literatures. Psychological Record, 1978, 28, 557-570.

Eiserrer, L. A., \& Hoffman, H. S. Priming of ducklings' responses by presenting an imprinted stimulus. Journal of Comparative and Physiological Psychology, 1973, 82, 345-359.

Eiserer, L. A., \& Hoffman, H. S. Acquisition of behavioral control by the auditory features of an imprinting object. Animal Learning \& Behavior, 1974, 2, 275-277.

Eiserer, L. A., Hoffman, H. S., \& Klein, S. H. Persistence of acquired behavioral control in the context of imprinting. Journal of the Experimental Analysis of Behavior, 1975, 24, 255-266.

Gaioni, S. J., Hoffman, H.S., DePaulo, P., \& Stratton, V. N. Imprinting in older ducklings: Some tests of a reinforcement model. Animal Learning \& Behavior, 1978, 6, 19-26.

Garcia, J., Kimeldorf, D. J., \& Koelling, R. A. A conditioned aversion towards saccharin resulting from exposure to gamma radiation. Science, 1955, 122, 157-158.

Graves, H. B. Early social responses in Gallus: A functional analysis. Science, 1973, 182, 937-939.

Gray, P. H. Evidence that retinal flicker is not a necessary condition of imprinting. Science, $1960,132,1834-1835$.

Hinde, R. A. The modifiability of instinctive behavior. Advancement of Science, London, 1955, 12, 19-24.

Hoffman, H. S., Barrett, J., Ratner, A. M., \& Singer, D. Conditioned suppression of distress calls in imprinted ducklings. Journal of Comparative and Physiological Psychology, 1972, 80, 357-364.

Hofrman, H. S., \& DePaulo, P. Behavioral control by an imprinting stimulus. American Scientist, 1977, 65, 58-66.

Hoffman, H. S., Eiserer, L. A., \& Singer, D. Acquisition of behavioral control by a stationary imprinting stimulus. Psychonomic Science, 1972, 26, 146-148.

Hoffman, H. S., \& Ratner, A. M. A reinforcement model of imprinting: Implications for socialization in monkeys and men. Psychological Review, 1973, 80, 527-544. (a)

Hoffman, H. S., \& Ratner, A. M. Effects of stimulus and environmental familiarity on visual imprinting in newly hatched ducklings. Journal of Comparative and Physiological Psychology, 1973, 83, 11-19. (b)

Hoffman, H. S., Ratner, A. M., \& Eiserer, L. A. Role of visual imprinting in the emergence of specific filial attachments in ducklings. Journal of Comparative and Physiological Psychology, 1972, 81, 399-409.

Hoffman, H. S., Ratner, A. M., Eiserer, L. A., \& Grossman, D. J. Aggressive behavior of immature ducklings. Journal of Comparative and Physiological Psychology, 1974, 86, 569-580.

Hoffman, H. S., Stratton, J. W., \& Newby, V. The control of feeding behavior by an imprinted stimulus. Journal of the Experimental A nalysis of Behavior, 1969, 12, 847-860.

Hoffman, H. S., Stratton, J. W., Newby, V., \& Barrett, J. E. Development of behavioral control by an imprinting stimulus. Journal of Comparative and Physiological Psychology, 1970, 71, 229-236.

Jonfs, R. B. Open-field responses of domestic chicks in the presence or absence of familiar cues. Behavioral Processes, $1977,2,315-323$.

Malcom, S. M., \& Graves, H. B. Responses of Gallus chicks to the static environment. Behavioral Biology, 1977, 21, 251-266. 
Pavlov, I. P. Conditioned reflexes. (G. V. Anrep, trans.) London: Oxford University Press, 1927.

Salzen, E. A. Imprinting and fear. Symposium of the Zoological Society of London, 1962, 8, 199-217.

Salzen, E. A. Imprinting and environmental learning. In L. R. Aronson, E. Tobach, D. S. Lehrman, \& J. S. Rosenblatt (Eds.), Development and evolution of behavior. San Francisco: Freeman, 1970.

Salzen, E. A., Lily, R. E., \& McKeown, J. R. Colour preference and imprinting in domestic chicks. Animal Behaviour, 1971, 19, 542-547.

Sluckin, W., \& Salzen, E. A. Imprinting and perceptual learning. Quarterly Journal of Experimental Psychology, 1961, 13, 65-77.
Taylor, A., \& TAYlor, K. R. Imprinting to a static feature of the home environment. Nature, 1964, 204, 1117-1118.

\section{NOTE}

1. Rather than attempt a precise definition of "conspicuous" in terms of some objective or physical characteristic, the present paper will follow the practice of Bateson $(1964 c)$ and-for the sake of expedience-use the word simply to describe stimuli as they appear to the human eye.

(Received for publicaton March 13, 1979; revision accepted August 22, 1979.) 